\title{
EVALUATING MANUFACTURING AND ASSEMBLY ERRORS IN ROTATING MACHINERY TO ENHANCE COMPONENT PERFORMANCE
}

\author{
Irem Y. Tumer* \\ Caelum Research \\ NASA Ames Research Center
}

\author{
Edward M. Huff \\ Computational Sciences \\ NASA Ames Research Center
}

\author{
"MS 269-3 \\ Moffett Field, CA 94035 \\ 650-604 2976 \\ itumer@mail.arc.nasa.gov
}

\begin{abstract}
Manufacturing and assembly phases play a crucial role in providing products that meet the strict functional specifications associated with rotating machinery components. The errors resulting during the manufacturing and assembly of such components are correlated with the vibration and noise emanating from the final system during its operational lifetime. Vibration and noise are especially unacceptable elements in high-risk systems such as helicopters, resulting in premature component degradation and an unsafe flying environment. In such applications, individual components often are subject to $100 \%$ inspection prior to assembly, as well as during operation through rigorous maintenance, resulting in increased product development cycles and high production and operation costs. In this work, we focus on providing designers and manufacturing engineers with a technique to evaluate vibration modes and levels for each component or subsystem prior to putting them into operation. This paper presents a preliminary investigation of the correlation between vibrations and manufacturing and assembly errors using an experimental test rig, which simulates a simple bearing and shaft arrangement. A factorial design is used to study the effects of: 1) different manufacturing instances; 2) different assembly instances; and, 3) varying shaft speeds. The results indicate a correlation between manufacturing or assembly errors and vibrations measured from accelerometers. Challenges in developing a tool for DFM are identified, followed by a discussion of future work, including a real-world application to helicopter transmission vibrations.
\end{abstract}

\section{MOTIVATION AND BACKGROUND}

The intended function of a component can be compromised if there are errors during production, which result in undesired side effects. Two of the significant factors that cause undesired vibrations are manufacturing and assembly errors. In this work, knowledge of the correlation between these errors and vibrations is seen as a crucial piece of information. To this end, we explore the relationship between vibration patterns in rotating machinery and manufacturing and assembly errors. A typical production cycle for rotating machinery components involves regular quality control steps to assure that the specifications are met satisfactorily. In particular, rotating components in high-risk applications such as rotorcraft transmissions are subject to an intense inspection process during manufacturing, before and after assembly, as well as during operation (maintenance). In such cases, $100 \%$ inspection of the parts' manufacturing and assembly tolerances are typically required, increasing the development time and the cost of producing such parts. A prediction of potential deviations from the intended functional requirements will not only reduce safety risks by avoiding premature failure, but also shorten the product cycle by 
avoiding scrap, rework, and inspection, as well as decrease costs associated with unplanned maintenance, hence reducing the overall cost of producing and operating such products (Tumer, Thompson et al. 1998; Tumer, Wood et al. 2000a; Tumer, Wood et al. 2000b).

\section{Tools for Design and Manufacturing}

Designers and manufacturing engineers use a combination of tools (six-sigma, inspection, statistical process control, Taguchi's robust design method, error budgeting, etc.) to assess and eliminate variation, with the goal of producing higher quality parts with less scrap or rework, hence, reducing the time and cost of product development (Bothe 1997; Carter 1997; Frey and Otto 1997; Bralla 1999; Chen and Thornton 1999; Tata and Thornton 1999). However, there is still a continuing need to develop products with better performance in faster and less costly ways. To address this problem, many researchers in design and manufacturing have been working towards developing tools to help designers and manufacturing engineers. The idea of predicting and controlling variation has resulted in several promising methods (Kazmer and Barkan 1996; Frey and Otto 1997; Chen and Thornton 1999; Suri and Otto 1999). Design tools that contain manufacturing variation information have been shown to facilitate the design process by reducing the number of iterations involved, estimating critical parameters, and identifying possible problems in terms of quality and cost (Bralla 1999).

In this work, the nature of the information passed onto the design and manufacturing engineer differs from standard "variation" information. Rather, the foundation of this work is based on the idea that the manufacturing process leaves a fingerprint on manufactured product surfaces, which can be investigated experimentally to determine the operating importance of such physical sources of variation. The underlying notion in this work is that such variations only become crucial when the performance of the system in which it is installed is directly affected by the nature and severity of these variations. Previous work focused on developing tools to extract this information in an accurate fashion, in order to help close the information gap between design and manufacturing (Tumer, Wood et al. 2000a; Tumer, Wood et al. 2000b). This paper extends the idea to the aerospace framework (Kromholtz and Condra 1993; Soni and Narang 1997), where the safe and cost-effective performance of rotating components is dependent on the quality of the products resulting from the manufacturing and assembly phases. The goal in this work is to develop practical tools to fit NASA's overall goal of designing and developing safer aircraft with shorter turn-around times, reduced cost, and increased quality.

\section{Characteristics of Ball Bearing Defects}

In this paper, ball bearings are selected as the initial focus of study because of their tendency to contain waviness and roughness errors on their inner and outer raceway surfaces, as well as their tendency to move within the bearing housing due to assembly errors. A defect-free ball bearing has perfectly circular inner and outer races, constant thrust loading, and no radial loads (Meyer, Ahlgren et al. 1980; Shigley and Mischke 1989). Any deviation from these intended functions is a defect. The most typical failures include point defects on the outer race, inner race, the rolling elements, and the cage: empirical formulas exist to compute the frequency at which these defects would appear in a vibration spectrum, usually a function of the shaft speed, ball diameter, number of balls, pitch diameter, and the contact angle (Braun 1980; Taylor 1980; Wowk 1991; Mitchell 1993). The relative amplitudes of these vibrational frequencies indicate the change in the bearing condition. 
Problems due to manufacturing and assembly errors are more difficult to isolate as single frequencies. Assembly errors are typically due to the bearing being mishandled after manufacturing, resulting in an improper mount or fit in the bearing housing for a system, or an improper mount or loading within the bearing cage. Such errors can result in looseness, unbalance, or misalignment, which are phenomena that typically result in high first, second, and third harmonics, as well as high harmonics (Wowk 1991; Brandlein, Eschmann et al. 1999). Manufacturing errors, defined as surface irregularities on bearing surfaces (typically under a micrometer in magnitude), are present in the form surface roughness and surface waviness (Braun and Datner 1979; Harris 1991; Su, Lin et al. 1993; Whitehouse 1994; Wensing and Van Nijen 1996; Ono and Okada 1998). Surface roughness patterns are high-frequency (noise-like) components, resulting in high-frequency components in the vibration spectrum. Waviness patterns are low-frequency components with a periodic pattern, resulting in low-frequency components in the vibration spectrum. Controlling component waviness and other types of errors from manufacturing, distortion, or damage occurring while the bearing is assembled to a machine, is a high priority, since the effects of such errors on machine vibration and noise can be significant (Harris 1991). Vibration testing and waviness testing are typically performed on components as part of quality control, but typically, overall levels and thresholds are used for determining the quality of such components (Harris 1991; Baldanzini, Beraldo et al. 1999; Chong, Yi et al. 1999).

\section{EXPERIMENTAL METHOD}

\section{Mechanical Apparatus}

The experimental test rig used in this experiment, the Machinery Fault Simulator (MFS), is shown in Figure 1. The product is currently marketed by SpectraQuest as a teaching aid, and was easily adapted for research use. As shown, the MFS is a desktop test rig, which houses a rotating shaft with weights to introduce balance faults, bearings attached at several locations to introduce bearing defects, and a gearbox attached to the main shaft by means of a belt to introduce gear defects. In the present study, most of the attachments provided with the device were taken off or disabled, such as the shaft unbalancing discs, belt drive to an external transmission assembly, and so forth, leaving the shaft free to rotate without load. This first set of experiments involves the motor main shaft, and the two ball bearing assemblies supporting the main shaft. Several types of bearings are provided for testing by the manufacturer, including a set of healthy and faulty ball bearings. In this study, three "normal" ball bearings were used for three production runs, making a total of nine different bearing/assembly combinations in the experiment.

\section{Data Collection Apparatus}

In order maintain consistency with vibration data collected on NASA test rigs and research aircraft, two single axis Endevco accelerometers were located near the bearing locations. During each experimental run, accelerometer and temperature sensors were sampled at $10 \mathrm{kHz}$ for 2.5 sec. using a data-acquisition system containing many of the same components used aboard the NASA test-rigs and research aircraft. The anti-aliasing filer was set at $5 \mathrm{kHz}$. The data 


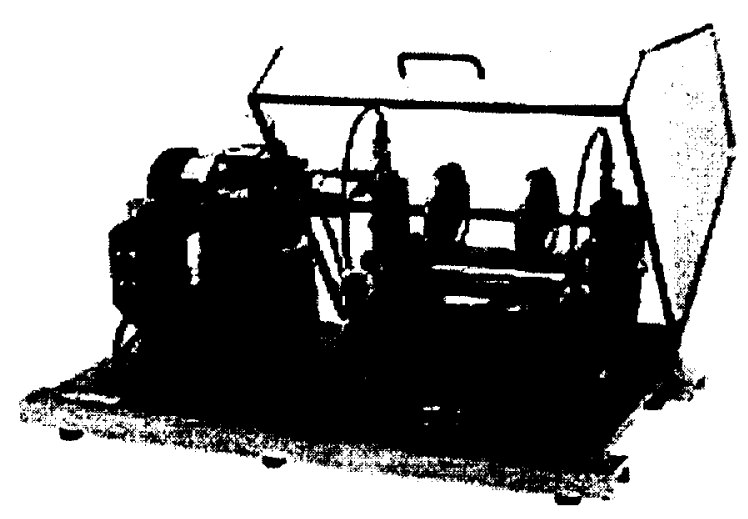

Figure 1. Mechanical Fault Simulator (MFS) manufactured by SpectraQuest.

collection process was controlled by a LabVIEW software package (ALBERT) specifically developed by NASA for test-rig operations (Huff, Tumer et al. 2000).

\section{Experimental Design and Analysis}

An experiment is designed using a factorial layout in order to test the effect of manufacturing and assembly errors, as well as shaft speed, and their interactions, on the vibration signature collected from the bearings. First, the signatures are compared to expected features from bearings, and analyzed using standard signal processing techniques, followed by an analysis of statistical differences between test conditions. Since the primary focus of the experiment was to examine the combined effects of manufacturing variations, assembly inaccuracies, and operating speed, a three-way factorial design was used which allows the assessment of interactions between the factors as well as their main effects using analysis-of-variance (ANOVA) techniques. Unusual aspects of this form of research, however, are that: (1) the dependent variables are time series rather than univariate measurements, and (2) the time series feature of greatest interest is total power, which, in the case of acceleration, represents the variance of the series. In other words, unlike typical empirical studies using ANOVA, this analysis is inherently concerned with a comparison of variance metrics rather than central tendencies. In order to meet the challenge this represented, an extrapolation was made to compare equality of treatment variances by use of the natural logarithm of the data (Montgomery 1991; Bendat and Piersol 1993).

In the present design, Factor A represents three fixed levels of shaft speed $(30 \mathrm{~Hz}, 45 \mathrm{~Hz}$, and $80 \mathrm{~Hz}$ ). Factor $B$ represents three assemblies of the bearings and is considered a random factor since we are interested primarily in generalizing to the population of assembly operations. With regard to Factor B, the three re-assemblies are conceptualized as simulating typical parts replacement procedures where the slightest misalignment, misfit, or looseness of the bearings in their housing can cause undesired vibrations that can be hazardous to the entire system's health. A rigorous re-assembly protocol was imposed to emulate the strict procedures followed when replacing helicopter transmission gear units (Huff, Tumer et al. 2000). Factor $\mathrm{C}$ represents the three bearing stocks from which three individual bearings were selected. Bearings from different stocks are assumed to contain manufacturing variations on their surfaces. This factor is also treated as a random factor since the population from which the stocks were drawn is of interest, not the particular three stocks themselves. The three factors with three levels each result in 27 
experimental test conditions. Each test condition is replicated 11 times to provide within cell variance for the ANOVA (Montgomery 1991).

\section{Computation of Defect Frequencies}

The bearings used for this study are SKF bearings with $\mathrm{N}=8$ balls, with a pitch diameter of $D_{m}=$ 1.1228 in. $(0.0285 \mathrm{~m})$, a ball diameter of $d=0.2813 \mathrm{in} .(0.0072 \mathrm{~m})$, and inner ring thickness of $t$ $=0.1083 \mathrm{in} .(0.0210 \mathrm{~m})$, an inner race circumference of $\mathrm{IR}=2.6437 \mathrm{in} .(0.0671 \mathrm{~m})$, and outer race circumference of $O R=5.0916$ in $(0.1293 \mathrm{~m})$. The defect frequencies can be computed as a function of the shaft rotational speed, which is at a frequency of 30,45 , or $80 \mathrm{~Hz}$, depending on the run condition, and a function of the ball diameter, pitch diameter, and number of balls. A simple geometry of a ball bearing is shown in Figure 1 for reference.

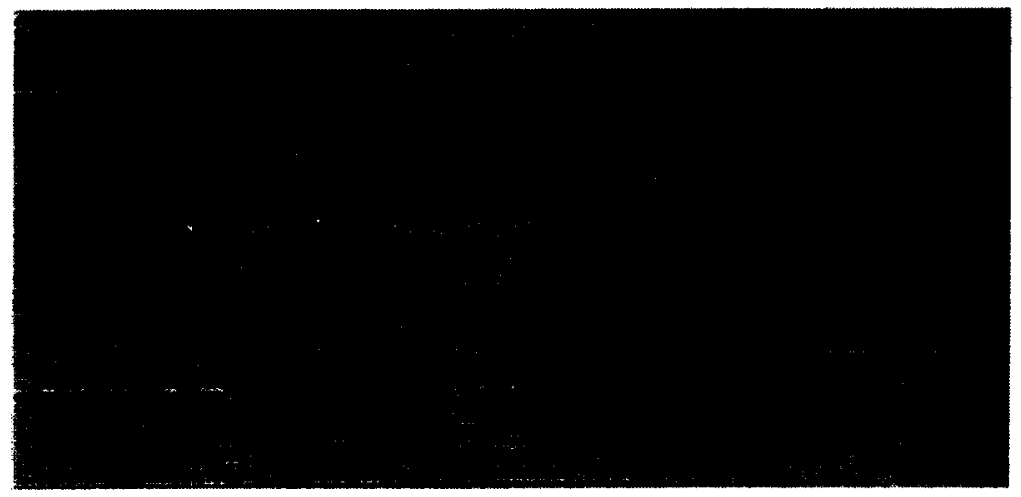

Figure 2. Ball Bearing Geometry.

A point defect on the outer race causes an impact each time a ball crosses it, resulting in $\mathbf{N}$ impacts for every revolution of the cage. Since the impacts are of short duration, the spectra will exhibit many harmonics. The cage frequency $f_{\text {cage }}$, and the corresponding outer race fault frequency $f_{\text {outerfault }}$ are shown in Table 1 for each shaft speed condition. A flaw on a single ball will alternately strike the inner and outer races, resulting in periodic forces at twice the ball spin frequency. Each impact is brief, and hence results in many harmonics. In addition, the contact points rotate at the cage precession frequency, which shows up as sidebands $f_{\text {cage }}$ apart. The ball spin frequency $f_{\text {ballspin }}$ and the corresponding ball fault frequency $f_{\text {ball fault, }}$, are also shown in Table 1. Finally, a flaw on the inner race will be impacted by each of the $\mathrm{N}$ balls in sequence, at an inner race contact frequency $f_{\mathbb{R} \text { contact, }}$ each will be brief and lead to harmonics. In addition, the fault location rotates with the shaft, resulting in sidebands $f_{\text {shaft }}$ apart. The resulting inner race fault frequency and the inner race contact frequency are shown in Table 1 for each speed.

The rotation of a ball with about its own axis is $f_{R}$, and the rotation of the inner race is $f_{\mathbb{R}}$. The vibration produced by waviness on the surface of a ball is computed as $f_{R}$ times the number of waves per ball circumference. The vibration produced by inner race waviness is computed as $f_{\mathbb{R}}$ times the number of waves per inner race circumference. Similarly, the vibration produced by outer race waviness is computed as the cage frequency $f_{\text {cage }}$ times the number of waves per outer race circumference. The assumption is that any ball rolls over all the waves in the outer raceway in one cage revolution, resulting in a ball passage frequency over an individual wave cycle on the outer race. Unfortunately, there is no way of knowing the total number of waves on the outer race, inner race, or the ball surfaces, unless detailed measurements of the surface profiles are 
collected from the dismantled bearings. As a result, until such a measurement is made, there are no reported values for these expected frequencies.

Table 1. Computed Defect Frequencies (all frequencies in $\mathrm{Hz}$; harmonics at $\mathrm{K}=1,2,3, \ldots$ )

\begin{tabular}{|c|c|c|c|c|c|c|}
\hline $\mathbf{f}_{\text {shant }}$ & $\mathbf{f}_{\text {cage }}$ & $\mathbf{f}_{\text {outerfaull }}$ & $\mathbf{f}_{\text {ballspin }}$ & $\mathbf{f}_{\text {ballfault }}$ & $\mathbf{f}_{\text {IRcontact }}$ & $\mathbf{f}_{\text {innerfault }}$ \\
\hline $30 \mathrm{~Hz}$ & 11.24 & $\mathrm{~K}^{*} 89.93$ & 56.11 & $\mathrm{~K}^{*} 112.2+-11.24$ & 18.76 & $\mathrm{~K}^{*} 150.0+-30$ \\
\hline $45 \mathrm{~Hz}$ & 16.86 & $\mathrm{~K}^{*} 134.9$ & 84.17 & $\mathrm{~K}^{*} 168.3+-16.86$ & 28.14 & $\mathrm{~K}^{*} 225.1+-45$ \\
\hline $80 \mathrm{~Hz}$ & 29.97 & $\mathrm{~K} * 239.8$ & 149.63 & $\mathrm{~K}^{*} 299.3+-29.98$ & 50.02 & $\mathrm{~K}^{*} 400.2+-80$ \\
\hline
\end{tabular}

\section{ANALYSIS OF RESULTS}

\section{Power Spectral Analysis of Vibration Data}

Before conducting a statistical analysis of the results, the vibration data are analyzed using standard signal processing techniques. The most standard means of analyzing vibration data is based on the power spectral analysis, which transforms the data into the frequency domain, isolating the frequency components of interest. The purpose is to investigate the frequency characteristics of the vibration data, and to isolate specific defect frequencies. Despite many problems faced by spectral analysis, especially in the presence of time-varying trends, to be compatible with industry standards, this paper uses the standard technique (Tumer, Wood et al. 2000a).

The experiments described above are conducted so that, for each bearing component, all three speeds and three assembly instances are tested. So, component 1 , for example, is the first bearing assembled into the bearing housing once, and used at speeds 1,2, and 3, and then disassembled, and reassembled into the bearing housing a second time, and run at speeds 1,2 , and 3 , and finally disassembled and reassembled a third time. Then component 1 is replaced with the second bearing component, and then replaced again with the third bearing component, and the same steps are followed to cover all 27 combinations of speeds, components, and assembly instances. As a result, for each component, the results are expected to be similar at the three speeds. The different bearings represent a random sample of manufacturing errors (waviness and/or roughness errors) on the outer race, inner race, or the ball surfaces. Similarly, point defects can be present either on the outer race, inner race, or ball surfaces. The power spectra should provide a picture of the frequency content of the vibration data, including the frequency components associated with the defects. One final note is on the assembly and component factors: because the assembly procedure is followed using strict steps, the only effects likely to be detected are due to uncontrollable factors, which may or may not be repeated in the experiments. As a result, every time a new bearing component is used, disassembled and reassembled, the experiment might be introducing effects that are not repeatable.

Figure 3 provides an overall picture of the frequency content for components 1, 2, and 3, assemblies 1,2 , and 3, for the low speed runs $(30 \mathrm{~Hz})$. The top three spectra correspond to the first bearing component, assembled into the same housing three times. The next three plots correspond to the second bearing component, and the last three correspond to the third bearing component. Similar plots are studied for each speed, and each replicate. Figure 4 presents a comparison of the frequency content for component 1 , assembly 1 , for each of the three speeds. An initial analysis of the power spectra indicates that the overall magnitudes of the frequency components are higher for component 2 than for component 1 . This effect is repeated over all the 
replicates, and speeds. In addition, the spectra for component 2 indicate the presence of higher frequency components, whereas the spectra for component 1 are cleaner in the higher frequencies. This pattern is indicative of a defect in component 2 , possibly in the form of surface waviness errors either on the outer race or the inner race. This conclusion will not be finalized until the inner and outer race surface profiles are measured and compared for the two bearings. Component 3 , on the other hand, implies a different set of conclusions. The magnitudes of a couple of the harmonics are much higher in comparison to the spectra for the first 2 components. In addition, a lot more variability is present between the different replicates, possibly indicating an assembly error within the bearing, since the frequency content is not repetitive or consistent.
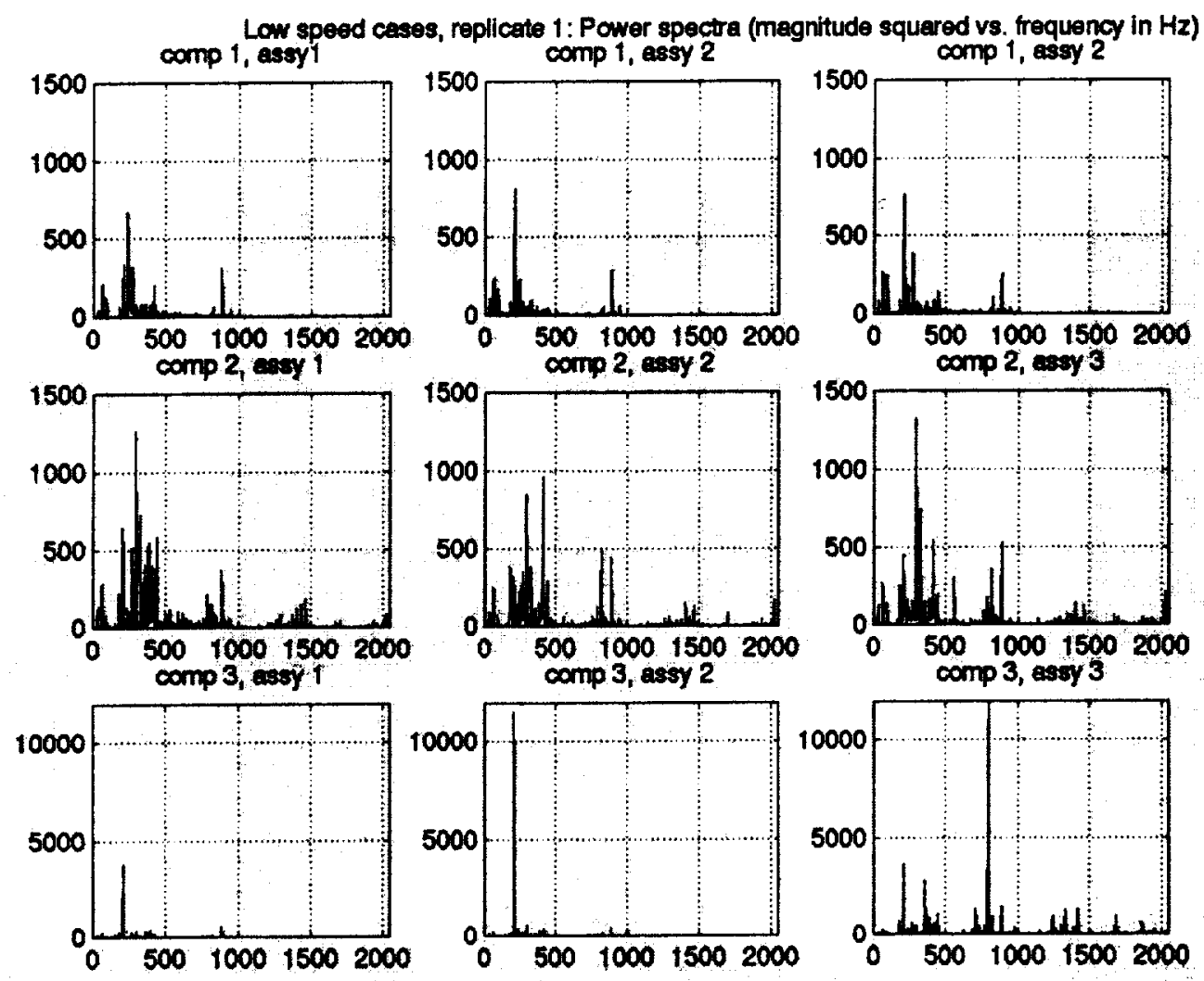

Figure 3. Power Spectral Analysis, Low-Speed Case, Replicate 1.

Overall, the spectra from all the cases display the typical frequency content for rotating machinery, composed of the shaft rotational frequency and its many harmonics. Figure 4 shows a comparison of the spectra for each of the three shaft speeds tests. Notice that the amplitudes of the frequency components increase as the shaft rotational speed increases. The first, second, and third plots correspond to shaft speeds of $30 \mathrm{~Hz}, 45 \mathrm{~Hz}$, and $80 \mathrm{~Hz}$, respectively, and show their corresponding harmonics. The presence of harmonics can also be indicative of potential misalignment or mechanical looseness problems, which can both be introduced due to assembly errors. In general, the magnitudes of these shaft harmonics dominate the spectra, possibly masking any of the smaller effects that would be indicative of defects. However, a detailed analysis of the spectra for low and medium speeds shows a couple of frequencies that are not multiples of the shaft frequency, corresponding to the fault frequencies computed in Table 1. Specifically, the low speed spectra show a frequency component in the surroundings of $90 \mathrm{~Hz}$ and 
another frequency component around $150 \mathrm{~Hz}$. Similarly, the medium speed spectra show additional frequency components in the surroundings of $135 \mathrm{~Hz}$ and $225 \mathrm{~Hz}$. From Table 1, the first outer race fault frequency should be at $89 \mathrm{~Hz}$ for the low speed runs, and $134 \mathrm{~Hz}$ for the medium speed runs; the first inner race fault frequency should be at $150 \mathrm{~Hz}$ for the low speed runs, and $225 \mathrm{~Hz}$ for the medium speed runs. Slight deviations from these computed frequencies are typically expected due to variations in speed, geometry, and other conditions that cannot be accounted for. The magnitudes of the shaft speed and their harmonics are too high to show any defect frequencies for the high-speed cases. In general, the spectra are dominated by the shaft speed and their harmonics, making it difficult to isolate defect frequencies in a conclusive matter.

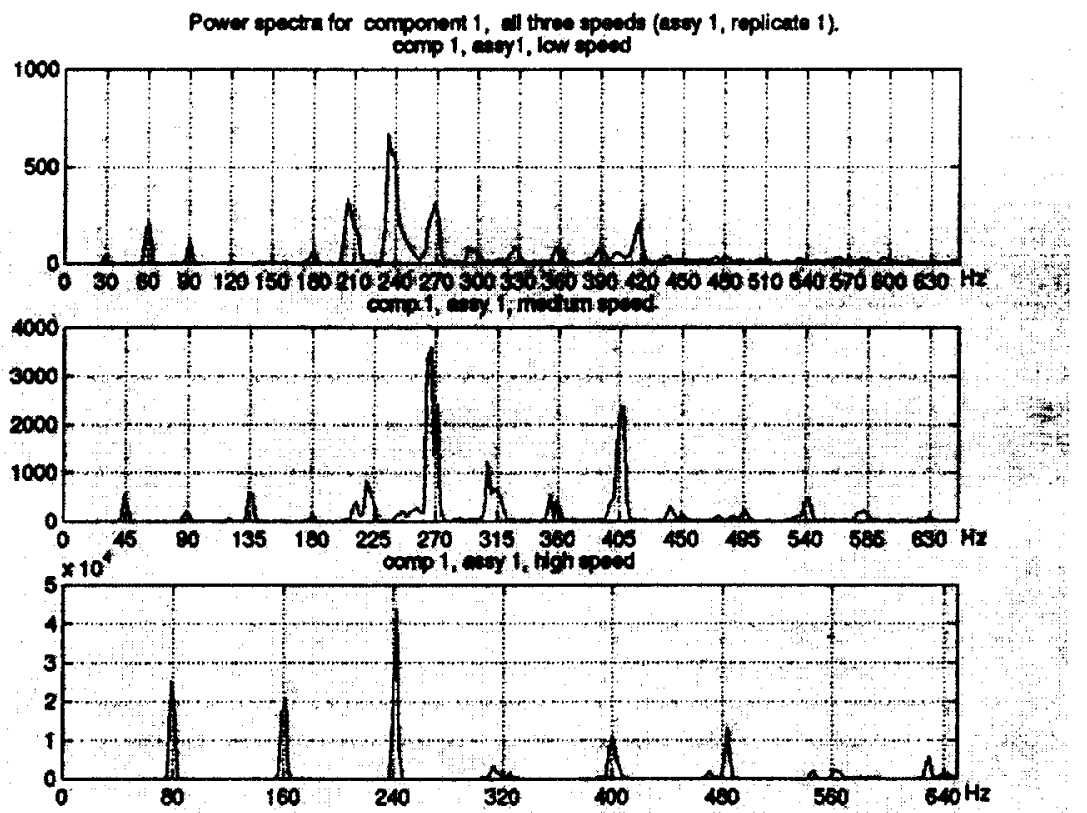

Figure 4. Power Spectra Comparison for Low, Medium, and High Speeds.

\section{Analysis of Changes in Total Power}

A general observation from the analysis above is that the total power in the power spectra varies for each of the bearing components, for each speed case, in a manner that is suggestive of some defects in the components. To investigate this observation further, the total power (area under the power spectrum) is analyzed for each test condition. The change in the total power for each component and assembly instances for the low, medium, and high speed cases is shown in Figures 5, 6, and 7 respectively. The $\mathrm{X}$-axis in these plots corresponds to each test run, for each component and assembly conditions, with 11 replicates for each run. For the case of the low speed runs, shown in Figure 5, component 1 shows the lowest total power levels, with a consistent increase for component 2 , which validates earlier observations from the power spectral analysis. Component 2 has some type of a defect that results in higher total power, possibly due to manufacturing errors. The total power levels are initially lower for component 3 than it was for component 2, but increase slightly for the rest of the runs. In particular, component 3 is shown in Figure 5 as manifesting a large amount of variability in the case of the third assembly. It is possible that an assembly error was introduced during the final disassembly and reassembly phase, hence resulting in the spectral energy to vary as the magnitude and direction of the impact 
forces on the bearings and shaft vary due to assembly errors. For the rest of the components, it is difficult to observe any changes due to the different assembly conditions.

Figure 6 shows the same plot for the medium speed case. In general, more variability is introduced amongst the replicates as the shaft speed in the experiments is increased. The total power for component 2 is slightly higher than for components 1 and 3 , just as was observed for the low speed runs. It is difficult to observe any differences due to the three assembly conditions. Finally, Figure 7 shows the same plot for the high-speed case. Note that the variability in the total power is so significant that it is difficult to isolate any specific patterns for the different bearing components.

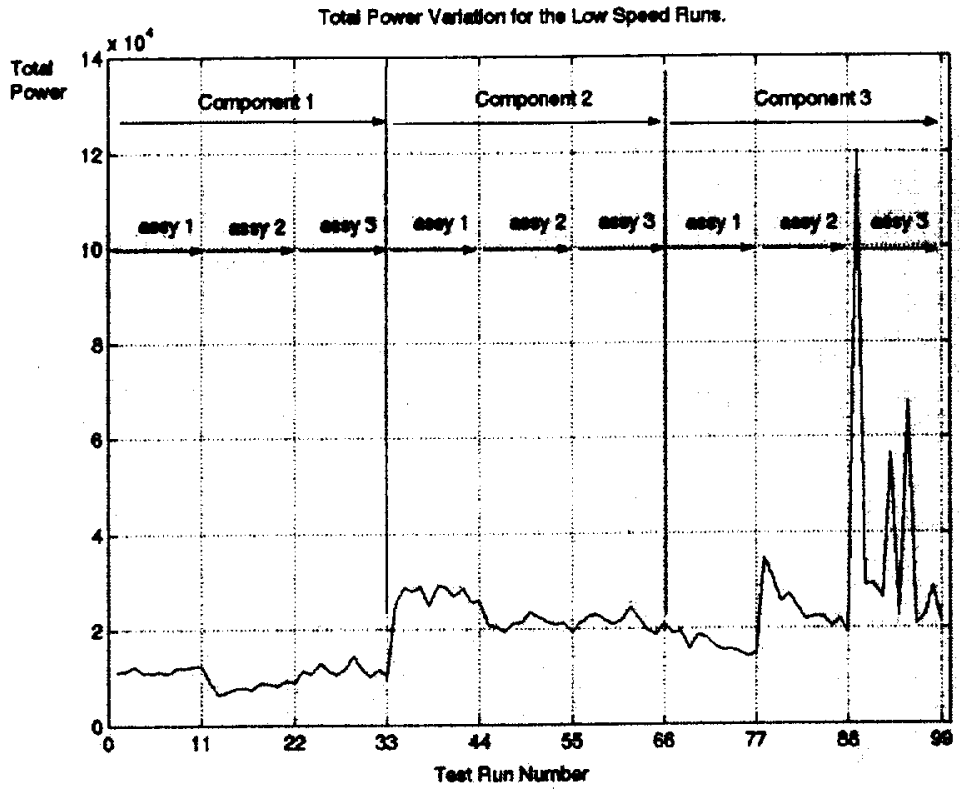

Figure 5. Total Power Variation for Low Speed Runs.

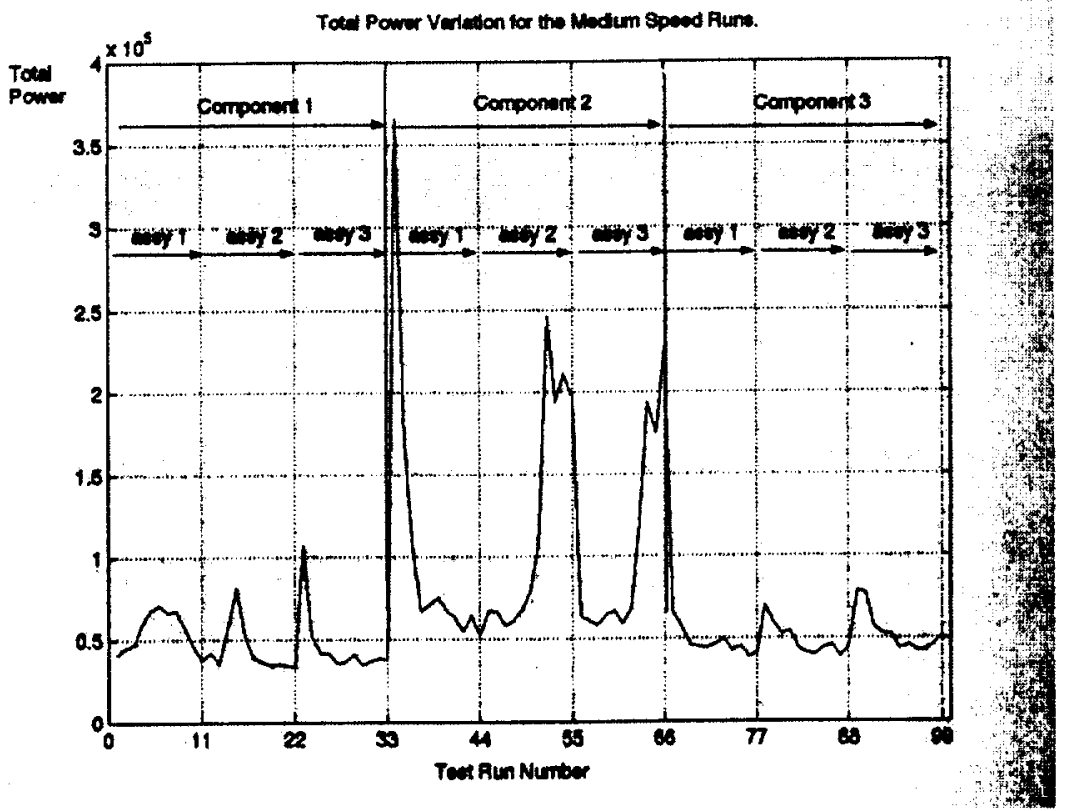

Figure 6. Total Power Variation for Medium Speed Runs. 


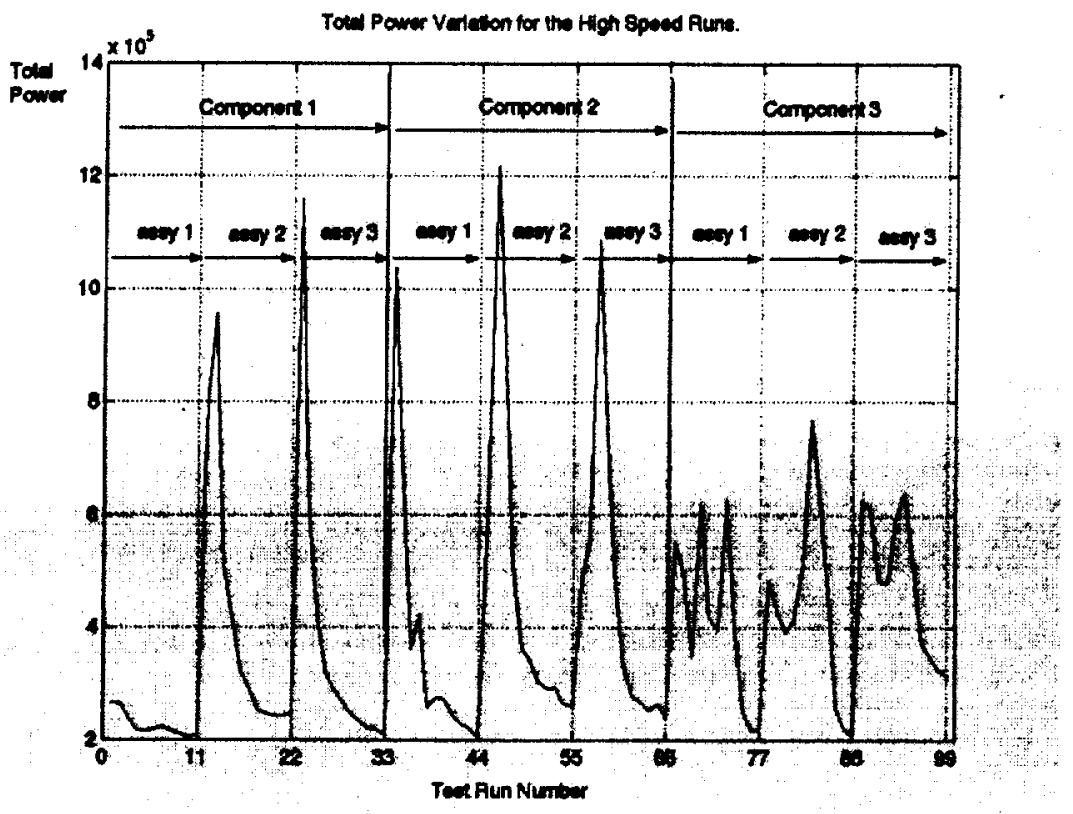

Figure 7. Total Power Variation for High Speed Runs.

\section{Analysis of Variance}

The analysis of the frequency content and total power for the test conditions shows some preliminary trends that indicate that manufacturing defects (represented in the form of three different bearing components) have an effect on the vibrational energy. Since speed is a major factor in the amount of vibrational energy emanating from the bearing fault simulator setup, it is reasonable to expect a second and third order interaction between speed and manufacturing errors, and possibly assembly errors. An analysis of variance approach is used to study these interaction effects, as well as the individual effects and their significance for the vibrational energy. The response metric is total power of the power spectra (which equals the variance of the vibration series in the time domain.) The main assumption for an ANOVA approach is that the data are sampled from a normal distribution (Montgomery 1991). Figure 8 shows a sample normal probability plot for the vibration data, indicating that the normality assumption is met for the originating data. In addition, the response metric used must also show normal characteristics. However, as observed from the analysis presented above, the total power varies greatly as a function of speed, hence casting some doubt to the validity of the results. A typical solution to such a problem is to transform the response metric to make it behave as a normal distribution. Figure 9 shows the normal probability plot for log-transformed total power.

To get a correct picture of the results, we present ANOVA results for the total power, and compare it to results for the log-transformed total power. Table 2 is an ANOVA table for total power based on a mixed-effects model (Montgomery 1991). SS is the sum of squared, df is the degrees of freedom, MS is the mean square value, $\mathrm{F}$ is the F-statistic value to assess significance, to be coupled with the significance level which indicates the probability that the effect is significant. 


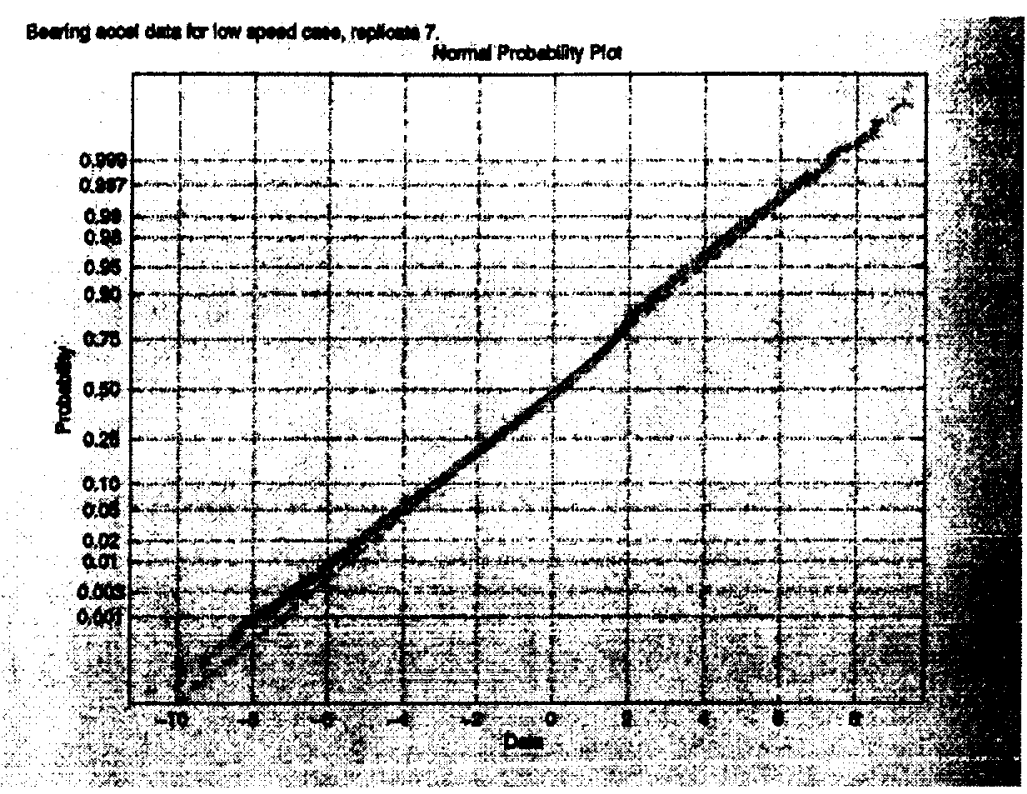

Figure 8. Normality Plots for Accelerometer Data.

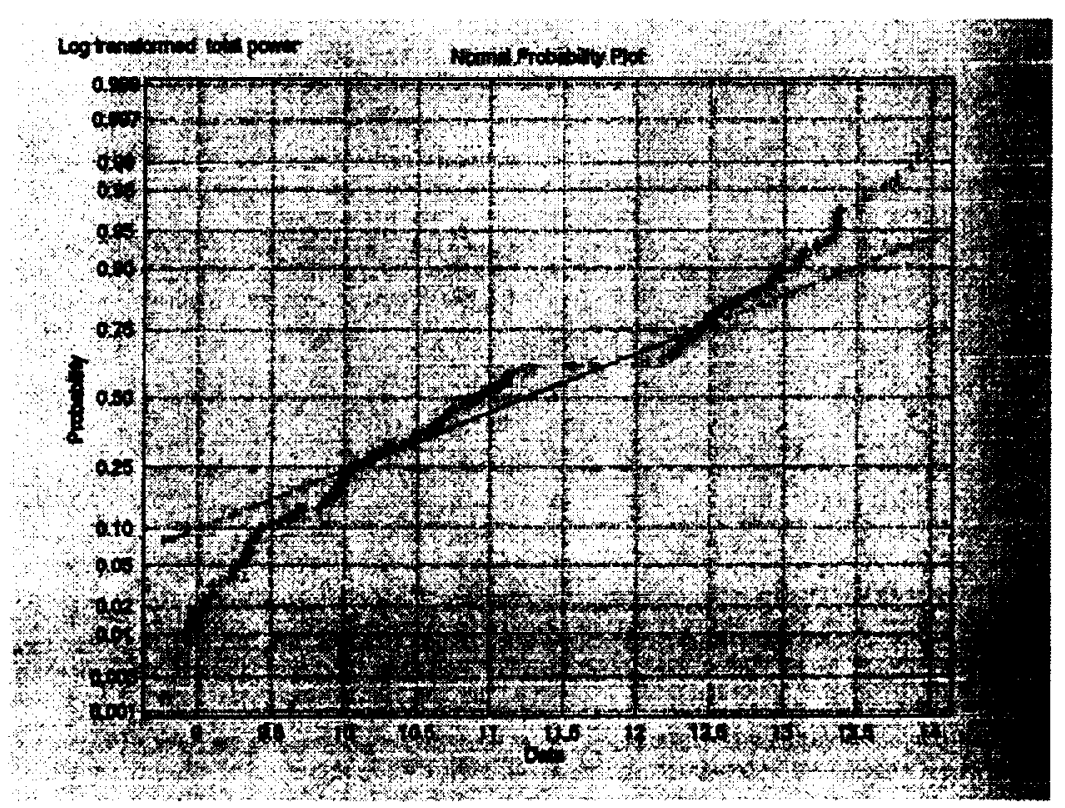

Figure 9. Normality Plots for Log Transformed Total Power.

The results from Table 2 show that speed is the main effect with a significance level corresponding to a perfect probability value $(0.000)$. The next two significant factors are the interactions speed has with assembly and manufacturing errors, significant with a probability level of 0.03 . Finally, manufacturing alone could be assessed as the fourth significant effect, if the probability level were set at 0.2 .

These initial results validate the general observations from the power spectral analysis. Going one step further, Table 3 shows the ANOVA results for the log-transformed total power metric, providing more reliable conclusions about the significance of each individual and interaction effect. The results from the transformed metric indicate that speed is the main effect with a 
Table 2. Analysis of Variance Results for Total Power.

\begin{tabular}{|c|c|c|c|c|c|}
\hline Source & SS & Df & MS & $\mathbf{F}$ & Sig. \\
\hline \multirow[t]{2}{*}{ Intercept } & $8.17 e+12$ & 1 & $8.17 e+12$ & 66.620 & .003 \\
\hline & $3.83 e+11$ & 3.126 & $1.22 \mathrm{e}+11$ & & \\
\hline \multirow[t]{2}{*}{ Speed } & $8.80 \mathrm{e}+12$ & 2 & $4.39 e+12$ & 70.047 & .000 \\
\hline & $3.90 \mathrm{e}+11$ & 6.217 & $6.28 \mathrm{e}+10$ & & \\
\hline \multirow[t]{2}{*}{ Assy } & $7.97 \mathrm{e}+10$ & 2 & $3.98 \mathrm{e}+10$ & 1.137 & .416 \\
\hline & $1.23 \mathrm{e}+11$ & 3.526 & $3.50 \mathrm{e}+10$ & & \\
\hline \multirow[t]{2}{*}{ Mfg } & $1.79 \mathrm{e}+11$ & 2 & $8.95 e+10$ & 2.693 & .196 \\
\hline & $1.61 \mathrm{e}+11$ & 3.492 & $3.32 e+10$ & & \\
\hline & $1.45 \mathrm{e}+11$ & 4 & $3.63 e+10$ & 4.554 & .033 \\
\hline $\begin{array}{r}\text { Speed*Assy (hyp) } \\
\text { Error }\end{array}$ & $6.37 e+10$ & 8 & $7.97 \mathrm{e}+09$ & & \\
\hline Speed*Mfg (hyp) & $1.37 \mathrm{e}+11$ & 4 & $3.44 \mathrm{e}+10$ & 4.325 & .037 \\
\hline \multirow{2}{*}{$\begin{array}{ll} & \text { Error } \\
\text { Assy*Mfg (hyp) }\end{array}$} & $6.37 e+10$ & 8 & $7.97 \mathrm{e}+09$ & & \\
\hline & $2.69 e+10$ & 4 & $6.73 e+09$ & .845 & .534 \\
\hline \multirow{2}{*}{$\begin{array}{r}\text { Error } \\
\text { Speed*Assy*Mfg }\end{array}$} & $6.37 e+10$ & 8 & $7.97 e+09$ & & \\
\hline & $6.37 e+10$ & 8 & $7.97 \mathrm{e}+09$ & .469 & .877 \\
\hline Error & $4.58 \mathrm{e}+12$ & 270 & $1.69 \mathrm{e}+10$ & & \\
\hline
\end{tabular}

probability of 0.001 , followed by the third order interaction between speed, manufacturing errors, and assembly errors (probability of 0.002 ). The second order interaction of speed and manufacturing is the third significant effect with a probability of 0.019 . Manufacturing errors could be assessed as significant, if the acceptable probability level were set at 0.2 (significance level of 0.117 ). Assembly errors do not show to be a significant factor by itself, or in its interactions with the other two factors. Recall that results from Table 2 indicated a possible interaction of the assembly factor with speed.

Table 3. Analysis of Variance Results for Log Transformed Total Power.

\begin{tabular}{|c|c|c|c|c|c|}
\hline Source & SS & df & MS & $\mathbf{F}$ & Sig. \\
\hline Intercept (hyp) & 37221.742 & 1 & 37221.742 & 4047.495 & .000 \\
\hline Error & 18.687 & 2.032 & 9.196 & & \\
\hline (hyp) & 454.178 & 2 & 227.089 & 101.344 & .001 \\
\hline Error & 8.314 & 3.710 & 2.241 & & \\
\hline (hyp) & .807 & 2 & .404 & 1.439 & .510 \\
\hline Error & .277 & .987 & .281 & & \\
\hline (hyp) & 18.224 & 2 & 9.112 & 4.154 & .117 \\
\hline Error & 7.843 & 3.576 & 2.193 & & \\
\hline Speed*Assy(hyp) & 1.467 & 4 & .367 & .904 & .505 \\
\hline Error & 3.245 & 8 & .406 & & \\
\hline Speed*Mfg (hyp) & 9.118 & 4 & 2.280 & 5.620 & .019 \\
\hline Error & 3.245 & 8 & .406 & & \\
\hline Assy*Mfg (hyp) & 1.277 & 4 & .319 & .787 & .565 \\
\hline Error & 3.245 & 8 & .406 & & \\
\hline Speed*Assy*Mfg & 3.245 & 8 & .406 & 3.188 & .002 \\
\hline Error & 34.352 & 270 & .127 & & \\
\hline
\end{tabular}




\section{DISCUSSION OF RESULTS AND FUTURE WORK}

The analysis of the vibration data discussed in the above sections point to an initial correlation between manufacturing defects and vibrational signature changes. Specifically, a random sample of bearings drawn from a population of bearings is assumed to contain manufacturing defects in the form of surface waviness errors. The results above have shown a possible manufacturing defect in the second bearing used for the experiments. The analysis of variance based on the experiments indicates that manufacturing errors and their interactions with the different shaft rotational speeds can have a relatively significant effect on the total power in the vibrational energy. While providing us with an initial empirical correlation between manufacturing errors and vibrational energy, the difficulty in analyzing the vibrational signatures for specific defects is clearly demonstrated throughout this paper. This difficulty presents a serious roadblock to the development of tools to assess the performance characteristics of rotating components, at the development stage. The accepted solution is to provide separate inspection procedures before and after a product is placed in operation: manufacturing tolerances (set based on experience) are used to inspect products after development; vibration thresholds (set based on experience) are used to test the products prior to sending them to their final destination. There are two problems with this approach: 1) it is costly and time-consuming; 2 ) it is not reliable enough. The first problem is common to all industry, lengthening the production time and cost considerably. The second problem requires continuous monitoring and inspection of individual components throughout their lifetime, particularly when such components are produced for high-risk applications such as aircraft. In this work, we are trying to address both problems by establishing a correlation between the errors during development and the degradation in the performance of rotating components. This paper showed a study to determine whether standard methods can be used to establish such a correlation, using empirical data, collected in a controlled test environment, using bearings as the main focus of the study.

Several important challenges were identified during this study. First, there is a lack of a reliable correlation between manufacturing errors and vibrational signatures, which makes it difficult to provide the correct information into the development cycle for early assessment of errors. Future work includes a measurement of the surface profiles of the bearings used in this study, as well as run tests with pre-fabricated manufacturing errors. Second, there is a lack of more accurate analysis methods to enable the detection of features that are not masked by other factors and features in the vibration data. Future work includes a search for more reliable tools to detect minute statistical differences indicative of manufacturing errors. Third, there is a clear difficulty in quantifying and assessing the effect of assembly errors on the vibration signature. Future work includes developing a quantifiable metric necessary to feed such information into the product development cycle.

The work and results described in this paper demonstrate the necessity and need of addressing the problem of correlating manufacturing and assembly errors with vibrations during operation.

Future work will concentrate on addressing the challenges identified above, so that automated tools can be developed to feed this information to the design and manufacturing engineers, in a systematic and reliable way. Currently, assembly errors are being studied in a separate experiment, using an $\mathrm{OH} 58$ helicopter transmission test rig at the NASA Glenn Research Center, with the purpose of investigating reinstallation effects on the main pinion housing vibrations (Huff, Tumer et al. 2000). Figure 10 shows a plot of the total vibrational power generated from this test rig. The experiment has three levels of torque, two levels of mast lifting forces, and two levels of mast bending forces. Phases $\mathrm{la}$ and $\mathrm{lb}$ correspond to the two phases before and after reinstallation; phase 1c corresponds to data collected after the first reinstallation, with a delay of several days; phase Id corresponds to data collected after the main pinion has gone through a 
second reassembly procedure. For the purposes of this paper, notice that the overall power levels show significant changes before and after reassembly, indicating the potential effect of assembly errors on the vibrational signature. Additional experiments are currently being planned using the MFS and the OH58 test rigs to address the rest of the challenges.

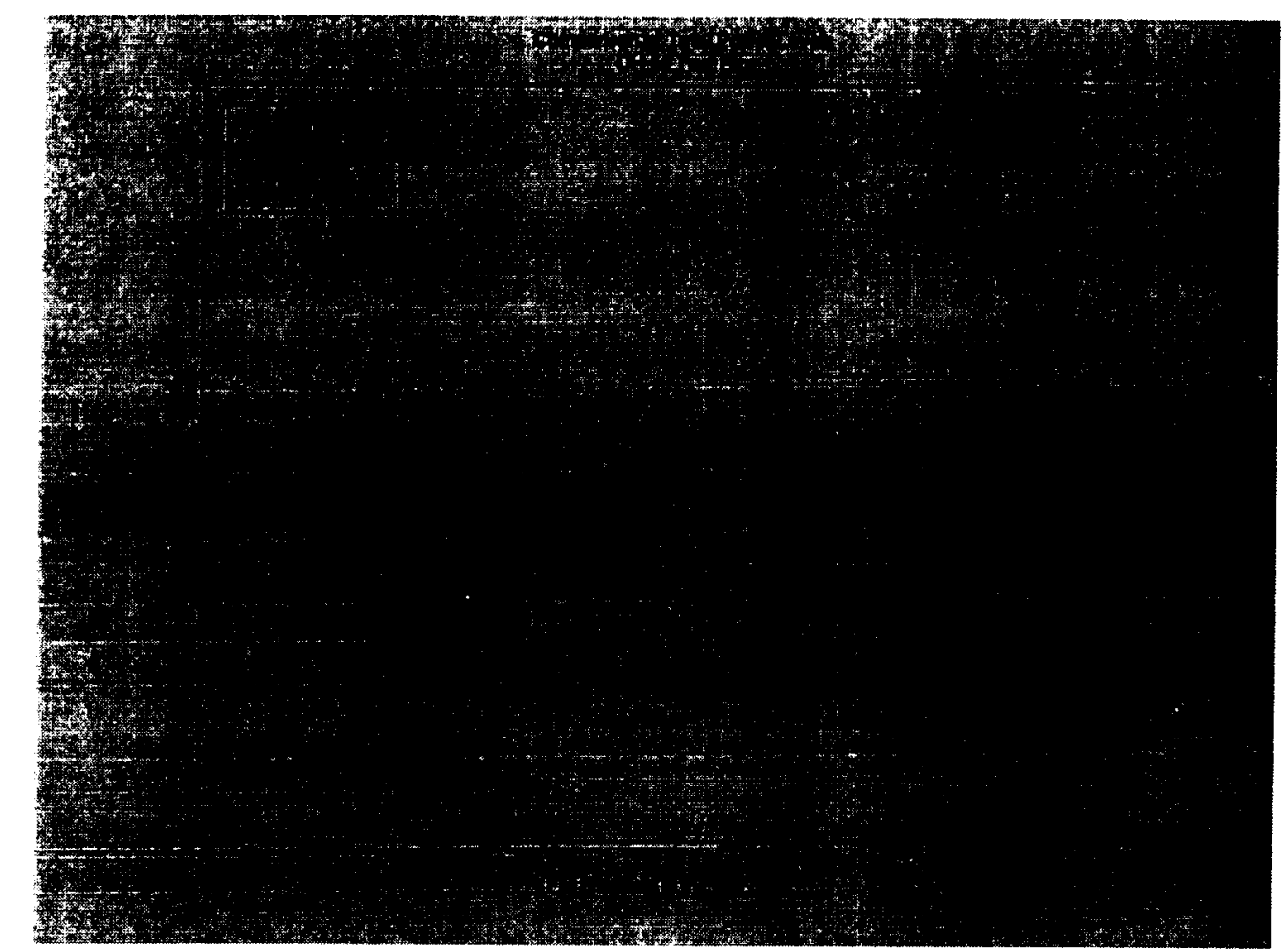

Figure 10. Comparison of Total Power Levels for an OH58 Transmission Test Rig.

\section{ACKNOWLEDGMENTS}

The authors would like to thank Eric Barszcz at NASA Ames for his help in running Albert; Mark Dzwonczyk, Alissa Fitzgerald, Larry Cochrane, and Tim Pfafman of Signal Processing Associates for their support on the data collection hardware; Dr. David Lewicki at NASA Glenn Research Center for his insights on manufacturing issues in helicopter applications; Dr. Surenda Ganeriwal and David Formenti at SpectraQuest for discussions about the Machinery Fault Simulator; and Jun Chen for his help in collecting the experimental data.

\section{REFERENCES}

Baldanzini, N., F. Beraldo, et al. (1999). Experimental Investigation of noise sources in a needle roller bearing system. ASME Design Engineering Technical Conferences, Las Vegas, NV. Bendat, J. S. and A. G. Piersol (1993). Engineering applications of correlation and spectral analysis, John Wiley and Sons.

Bothe, D. R. (1997). Measuring process capability: techniques and calculations for quality and manufacturing engineers, McGraw-Hill.

Bralla, J. G. (1999). Design for manufacturability handbook, McGraw-Hill.

Brandlein, J., P. Eschmann, et al. (1999). Ball and Roller Bearings: Theory, Design, and

Application, John Wiley and Sons.

Braun, S. and B. Datner (1979). "Analysis of Roller/Ball bearing vibrations." Transactions of the ASME 101(January): 118-125. 
Braun, S. G. (1980). "The signature analysis of sonic bearing vibrations." Transactions on Sonics and Ultrasonics Su-27(6): 317-328.

Carter, A. D. S. (1997). Mechanical Reliability and Design, John Wiley and Sons.

Chen, T. J. and A. C. Thornton (1999). Quantitative selection of inspection plans. ASME Design Engineering Technical Conferences, Las Vegas, NV.

Chong, A., L. Yi, et al. (1999). Experimental characterization techniques for high performance hard disk drive ball bearing spindle motors. ASME Design Engineering Technical conferences, Las Vegas, NV.

Frey, D. and K. Otto (1997). The process capability matrix: a tool for manufacturing variation analysis at the systems level. ASME Design Theory and Methodology Conference, Sacramento, CA.

Harris, T. A. (1991). Rolling bearing analysis, John Wiley and Sons.

Huff, E. M., I. Y. Tumer, et al. (2000). Experimental analysis of mast lifting and bending forces on vibration patterns before and after pinion reinstallation in an OH58 transmission test rig. American Helicopter Society Annual Forum, Virginia Beach, VA.

Kazmer, D. and P. Barkan (1996). Quantifying design and manufacturing robustness through stochastic optimization. ASME Design Automation Conference, Irvine, CA.

Kromholtz, G. A. and L. W. Condra (1993). "New approach to reliability of commercial and military aerospace products: beyond military quality/reliability standards." Quality and Reliability Engineering International 9(3): 211-215.

Meyer, L. D., F. F. Ahlgren, et al. (1980). "An analytic model for ball bearing vibrations to predict vibration response to distributed defects." Journal of Mechanical Design 102(April): 205210.

Mitchell, J. S. (1993). Introduction to machinery analysis and monitoring, PennWell Books. Montgomery, D. C. (1991). Design and Analysis of Experiments, John Wiley and Sons. Ono, K. and Y. Okada (1998). "Analysis of ball bearing vibrations caused by outer race waviness." Journal of Vibration and Acoustics 120: 901-908.

Shigley, J. E. and C. L. Mischke (1989). Mechanical engineering design, Mc-Graw-Hill, Inc. Soni, J. S. and S. C. Narang (1997). "Quality engineering in aerospace technologies: a review." Defence Science Journal 47(97): 5-18.

Su, Y. T., M. H. Lin, et al. (1993). "The effects of surface irregularities on roller bearing vibrations." Journal of Sound and Vibration 165(3): 455-466.

Suri, R. and K. Otto (1999). System-level robustness through integrated modeling. ASME Design engineering technical conferences, Las Vegas, NV.

Tata, M. and A. C. Thomton (1999). Process capability database usage in industry: myth vs. reality. ASME Design engineering technical conferences, Las Vegas, NV.

Taylor, J. I. (1980). "Identification of Bearing Defects by Spectral Analysis." Joumal of Mechanical Design 102(April): 199-204.

Tumer, I. Y., D. Thompson, et al. (1998). "Characterization of surface fault patterns with application to a layered manufacturing process." Journal of Manufacturing Systems 17(1): 23-36. Tumer, I. Y., K. L. Wood, et al. (2000a). "A mathematical transform to analyze part surface quality in manufacturing." ASME Joumal of Manufacturing Science and Engineering 122(February): 1-7.

Tumer, I. Y., K. L. Wood, et al. (2000b). "Monitoring of signals from manufacturing processes using the Karhunen-Loeve transform." Mechanical Systems and Signal Processing (accepted September 1999.).

Wensing, J. A. and G. C. Van Nijen (1996). 2-dimensional computational model for vibration analysis of waviness in rolling bearing applications. Sixth international conference on vibrations in rotating machinery.

Whitehouse, D. J. (1994). Handbook of surface metrology, Institute of Physics Publishing.

Wowk, V. (1991). Machinery vibration: measurement and analysis, McGraw-Hill. 